\title{
Frontières
}

\section{Sept interdits musulmans concernant des rites de deuil et autres rites entourant la mort Analyse historico-comparative}

\section{Jean-Jacques Lavoie}

Volume 24, numéro 1-2, automne 2011, printemps 2012

URI : https://id.erudit.org/iderudit/1013086ar

DOI : https://doi.org/10.7202/1013086ar

Aller au sommaire du numéro

Éditeur(s)

Université du Québec à Montréal

ISSN

1916-0976 (numérique)

Découvrir la revue

Citer cet article

Lavoie, J.-J. (2011). Sept interdits musulmans concernant des rites de deuil et autres rites entourant la mort : analyse historico-comparative. Frontières, 24(1-2), 63-72. https://doi.org/10.7202/1013086ar

\section{Résumé de l'article}

Dans cet article, l'auteur examine sept interdits musulmans à propos des rites autour de la mort : déchirer (l'encolure de) ses vêtements, se frapper, se raser la tête, se mettre de la poussière sur la tête, se lamenter, retarder l'enterrement et rester debout lorsque passe un convoi funèbre. Par le biais d'une analyse comparée, l'auteur dégage ensuite quelques dissimilitudes et similitudes significatives entre les règles provenant des hadiths de l'islam sunnite et celles du judaïsme rabbinique. 


\section{Résumé}

Dans cet article, l'auteur examine sept interdits musulmans à propos des rites autour de la mort: déchirer (I'encolure de) ses vêtements, se frapper, se raser la tête, se mettre de la poussière sur la tête, se lamenter, retarder l'enterrement et rester debout lorsque passe un convoi funèbre. Par le biais d'une analyse comparée, l'auteur dégage ensuite quelques dissimilitudes et similitudes significatives entre les règles provenant des hadiths de l'islam sunnite et celles du judaïsme rabbinique.

Mots clés: rites de deuil - islam judaïsme - analyse comparée.

\begin{abstract}
In this article, the author examines seven banned Muslim rituals concerning death: tearing (the neckline of) his clothes, beating of one's self, shaving their heads, placing dust on their heads, lamenting, delaying the funeral and standing when a funeral procession passes. Through a comparative analysis, the author then sets out some significant similarities and dissimilarities between the rules from the hadiths of Sunni Islam and those of rabbinic Judaism.
\end{abstract}

Keywords: mourning rites - islam judaism - comparative analysis.

\title{
Sept interdits musulmans concernant des rites de deuil et autres rites entourant la mort
}

\author{
Analyse historico-comparative
}

\author{
Jean-Jacques Lavoie, Ph. D. \\ professeur titulaire, Département de sciences \\ des religions, UQAM.
}

Maints travaux d'anthropologues et d'historiens illustrent avec éloquence la permanence des rites autour de la mort dans l'histoire de l'humanité. Encore de nos jours, en Amérique du Nord comme ailleurs, même si d'aucuns adoptent une anthropologie qui réifie le corps et réduisent la mort à un processus biologique, les rites de deuil et autres rites entourant la mort semblent être ceux qui résistent le plus à la sécularisation. De tout temps, les êtres humains, peu importe qu'ils aient été croyants, athées ou agnostiques, ont donc adopté certains rites à l'égard d'un proche qui meurt. Par contre, comme le rite est un agir social spécifique, programmé, répétitif et symbolique, par lequel s'opère l'identification de l'individu dans son groupe social et de ce groupe dans la société globale, les rites autour de la mort varient forcément selon les groupes sociaux, mais aussi les groupes religieux. C'est ce que l'on peut observer lorsque l'on examine les règles relatives aux rites autour de la mort dans le monde musulman. Plusieurs d'entre elles visent à distinguer l'islam des autres groupes religieux et parfois plus particulièrement du judaïsme. Cette distinction prend habituellement la forme d'un interdit. Or, ce sont quelques-uns de ces interdits que je compte examiner dans le présent article.

Pour comprendre la raison de ces interdits dans l'islam, je ferai appel à l'histoire comparée des religions. Il est bien connu, pour reprendre la célèbre affirmation de Müller à propos des religions, que celui qui «n'en connaît qu'une n'en connaît aucune» (Müller 1873, 16). En effet, une analyse comparée avec les textes de la tradition juive et même les textes du Proche-Orient ancien me permettra de découvrir la symbolique de quelques rites interdits par l'islam. Par le fait même, cette analyse comparée me permettra de mieux cerner la spécificité de l'islam, particulièrement à l'égard du judaïsme.

Mon objectif sera ici doublement limité. Premièrement, je n'examinerai que sept interdits musulmans à propos des rites autour de la mort. Mon enquête sera donc divisée en sept parties de longueur inégale, chacune portant sur un interdit particulier: déchirer (l'enco- 
lure de) ses vêtements, se frapper, se raser la tête, se mettre de la poussière sur la tête, se lamenter, retarder l'enterrement et rester debout lorsque passe un convoi funèbre.

Deuxièmement, mon analyse comparée portera sur un corpus limité, mais néanmoins bien représentatif de l'islam sunnite et du judaïsme rabbinique. En ce qui concerne le judaïsme rabbinique, deux traités constitueront mes principales sources d'information: le traité Śemāhôt (à l'avenir Sem) et le traité Mô‘ēd Qātān du Talmud de Babylone (à l'avenir MQ). Bien entendu, je prendrai également en considération le patrimoine littéraire juif qui précède l'époque de ces deux traités rabbiniques, c'est-à-dire le Tnak ou la Bible hébraïque, la littérature judéo-hellénistique de la période du Second Temple et la littérature intertestamentaire. Du côté de l'islam, je m'intéresserai essentiellement à la tradition rapportant les actes et les paroles du Prophète. Deux recueils de hadiths retiendront plus particulièrement mon attention: celui de Bukhârî (à l'avenir B) et celui d'Abû Dâwûd (à l'avenir AD), deux compilateurs qui ont vécu au $3^{\mathrm{e}}$ siècle de l'Hégire, c'est-à-dire au $9^{\mathrm{e}}$ siècle de l'ère chrétienne. Bien entendu, je n'hésiterai pas à faire appel à d'autres sources lorsque je le jugerai nécessaire.

Ce choix m'oblige d'entrée de jeu à reconnaître que je n'ai pas l'ambition d'être exhaustif et, par conséquent, que je ne prétends aucunement apporter ici des réflexions définitives. Toutefois, même s'il s'agit d'une étude exploratoire, le choix de mon corpus de textes n'a rien d'arbitraire. En effet, il est bien connu que le Coran ne donne guère de directives en ce qui concerne les rites autour de la mort. Seule l'inhumation du mort est évoquée (Lavoie, 2011 : 91-113). Les premiers musulmans devront attendre la mise par écrit des hadiths, qui constituent la deuxième source de la $\operatorname{sharī'}^{\prime} a$ après le Coran, pour avoir certaines consignes précises sur ce qu'il faut faire et surtout ne pas faire lorsqu'une personne meurt. Du côté juif, il est également bien connu que le $\mathrm{T}^{\mathrm{e}} \mathrm{nak}$, première source de la loi juive, ne comprend aucun traité sur les rites de deuil et rites funèbres. Dans le Talmud, on trouve de nombreuses informations sur ces rites dans le MQ. Par contre, le seul traité exclusivement réservé aux règles relatives aux rites autour de la mort est un traité para-talmudique connu sous le nom de $S^{e} m a \bar{h} h \hat{o} t$, littéralement « Joies», nom donné par euphémisme à ce qui devait s'appeler 'Ébel Rabbātî, «Le grand deuil». La datation de ce dernier traité ne fait pas l'unanimité - les spécialistes croient que la rédaction de Sem s'étend du $3^{\mathrm{e}}$ au $8^{\mathrm{e}}$ siècle de l'ère chrétienne (Cohn-Sherbof, 1992, p. 146; Walfish, 1997, p. 240) -, mais il est certain que les textes juifs que j'analyserai ont été rédigé avant les hadiths. Bien entendu, les textes juifs aussi bien que les textes musulmans reflètent des pratiques funéraires plus anciennes que leur époque de rédaction. C'est d'ailleurs ce dont témoigne la vaste littérature sémitique du Proche-Orient ancien, qui retiendra également mon attention.

Malgré la double limite de mon enquête limite au niveau du corpus et des interdits étudiés -, celle-ci sera doublement originale, d'une part, parce que je ferai appel à une analyse comparative avec des textes du monde juif et du Proche-Orient ancien et, d'autre part, parce que les sept interdits retenus n'ont guère fait l'objet d'une analyse approfondie de la part des islamologues. En effet, des sept interdits que je vais analyser, seul l'interdit de la lamentation, des cris et des pleurs a véritablement retenu l'attention de l'étude magistrale de Grütter (1954, p. 153; 158-161；1957, p. 100) à laquelle les islamologues qui s'intéressent aux rites autour de la mort renvoient sans cesse (Tritton, 1977, p. 454; Leisten, 1990, p. 19, note 4; Rāgib, 1992, p. 393; Zaman, 2001, p. 28; Halevi, 2007, p. 5 et 246 note 7). Autrement, elle ne réserve que quelques lignes aux quatre premiers interdits (1954: 154-155) et ne donne que quelques informations relatives aux deux derniers interdits (1957: 98-99). Qui plus est, l'enquête de Grütter est purement descriptive; l'auteure ne s'intéresse donc jamais à la symbolique de ces rites et aux raisons profondes qui ont pu motiver leur interdiction. En ce qui concerne l'excellent livre de Halevi, il ne contient qu'un chapitre sur les lamentations pour les morts (2007: 114-142). Le reste de sa brillante étude porte plutôt sur des sujets comme la pierre tombale, le linceul, la toilette funèbre, la construction des tombes, etc., des sujets certes très intéressants mais qui ne retiendront pas mon attention. Quant aux trois autres articles, ils traitent de sujets qui ne retiendront pas davantage mon attention: l'architecture funéraire (Leisten, 1990), la structure de la tombe (Rāgib, 1992), les controverses juridiques entre musulmans au sujet des funérailles et les récits relatifs aux funérailles provenant des biographies de quelques musulmans célèbres (Zaman, 2001).

\section{L'INTERDIT DE DÉCHIRER (L'ENCOLURE DE) SES VÊTEMENTS}

Le traité Sem mentionne à quelques reprises le rite de la déchirure des vêtements, rite connu sous le nom de $q^{e} r \hat{\imath}^{\prime} \bar{a} h$ :

le jour où l'on entend la mort est comme celui de son enterrement ( $q^{e}$ bûrāh $)$, autant que déchirer son vêtement

(liq $\left.{ }^{e} \hat{\imath} \hat{a}^{\prime} h\right)$ et prendre le deuil, le précepte concernant les sept jours et les préceptes concernant les trente jours doivent être respectés. (Sem 12,1)

Selon Sem 12,3, ce rite doit être pratiqué à deux moments distincts: "Ainsi que l'on déchire ses vêtements (̌́eqôr ${ }^{e}$ ' $\hat{i n}$ ) à la mort de quelqu'un, on doit aussi les déchirer (qô $r^{e}$ 'în) le jour où on rassemble les os» (voir aussi
Sem 1,5 et $8,8-9 ; 9,1.16-17$ et MQ 25a qui ne mentionne que le moment du décès). Ce rite ne doit toutefois pas être pratiqué par tous, mais seulement par les proches parents ou les élèves du maître:

Il ne faut pas déchirer ses vêtements (qôrē'a) à la mort de tous ceux qui ne sont pas proches parents, sauf dans le cas de son maître qui lui avait appris la sagesse. Rabbi Siméon ben Éléazar a dit: Si un savant meurt, tous ceux qui sont là au moment de sa mort doivent déchirer leurs vêtements $\left(q \hat{o} r^{e} \hat{i n}\right)$; tous ceux qui ne sont pas là n'ont pas besoin de déchirer leurs vêtements (qôor $\left.{ }^{e} \hat{i n}\right)$ pour

lui. (Sem 9,1; pour d'autres précisions, voir MQ 20b.)

Ce rite est interdit aux endeuillés si la personne qui est morte s'est suicidée (Sem 2,1). En ce qui concerne les mineurs, leurs vêtements doivent être déchirés par d'autres (Sem 9,6; MQ 14b.26b). Pour être valide, la déchirure doit se faire au niveau de l'encolure du vêtement:

Pour tous les autres morts, il n'a pas besoin de couper au niveau du col du vêtement (mab dîl qamî śāpāh); pour son père et sa mère, il doit couper au niveau du col du vêtement (mabe dîl qamî śāpāh). Rabbi Judah a dit: toute déchirure

$\left(q^{e} r a^{\prime}\right)$ qui n'est pas au niveau du col du vêtement (mab dîl qamî śāpāh) est une déchirure $\left(q^{e} r a^{\prime}\right)$ inutile ( $\left.t \hat{p}^{e} l \bar{a} h\right)$. (Sem

9,6)

Le mot tîp $p^{e} l \bar{a} h$, rendu ici par «inutile», signifie également «obscène». Si l'on retient ce second sens, il est clair que la déchirure ne doit pas permettre d'exhiber le corps nu. Sem 9,18 apporte une précision qui indique que les deux sens sont possibles: «Un vêtement doit être déchiré seulement entre les deux coutures du cou (bēenn šetēy hwmșywt). Quiconque déchire la partie inférieure ou sur les côtés n'a pas accompli son obligation. » Le vêtement qui doit être déchiré ne fait pas l'unanimité: «L'obligation de déchirer son vêtement est accomplie sans avoir à toucher le vêtement intérieur ('ip eyqareysîn). Rabbi Benjamin affirme au nom de Rabbi 'Aqiba: le vêtement intérieur ('ip ${ }^{e} y q a r^{e} y s i n$ ) doit être déchiré» (Sem 9,6; avec des variantes, voir aussi MQ 22b). La règle varie aussi selon qu'il s'agit d'un homme ou d'une femme (Sem 9,7 et MQ 22b). Quant à la réparation du vêtement déchiré, elle fait aussi l'objet de diverses règlementations très strictes (Sem 9,8.19; voir aussi MQ 22b.26a).

Le rite qui consiste à déchirer ses vêtements est très ancien. À l'origine, cependant, on déchirait ses vêtements à l'occasion autant d'un malheur en signe de lamentation que d'un décès en signe de deuil. Danel, roi mythique d'Ougarit, déchire son habit en signe de deuil (Légende d'Aqhat C 1, 38-49). Gilgamesh déchire aussi ses vêtements (7,12 selon le 
fragment de Megiddo) ou les rejette en signe de deuil $(8,2,22$, selon la version assyrienne). Selon Diodore de Sicile (Livre 1,72), lorsqu'un des rois d'Égypte venait à mourir, tous les habitants prenaient le deuil et déchiraient leurs vêtements. Ce rite de la déchirure du vêtement est largement attesté dans les trois parties de la Bible hébraïque ${ }^{1}$, dans les textes judéohellénistiques ${ }^{2}$, dans la littérature intertestamentaire $^{3}$ et dans le Nouveau Testament ${ }^{4}$.

Bien entendu, ce rite de la déchirure du vêtement est une façon d'exprimer l'idée que la mort est un déchirement qui provoque une intense souffrance. Par ailleurs, en permettant de retourner la violence contre soi, ce rite exprime aussi une colère qui introduit la mort dans la vie. Plus encore, l'acte de déchirer ses vêtements est un rite qui mime en quelque sorte la mort. Plusieurs arguments justifient cette interprétation. Premièrement, c'est ce que nous rappelle la comparaison de Sem 12,1 que j'ai déjà cité ci-dessus : «le jour où l'on entend la mort est comme celui de son enterrement ( $\left.q^{e} b \hat{u} r \bar{a} h\right)$, autant que déchirer son vêtement (liq $\left.{ }^{e} r \hat{\imath} ' \bar{a} h\right)$ et prendre le deuil»».

Deuxièmement, il est bien connu que le vêtement est à ce point en relation intime avec celui qui le porte qu'il peut être considéré comme un substitut de la personne. Par exemple, en Mésopotamie ancienne, certains rituels de guérison étaient des rituels où le substitut (très souvent un animal) était habillé avec les vêtements du malade (Cassin, 1968: 61). Toujours en Mésopotamie ancienne, lors de la fête du Nouvel An, comme le roi ne pouvait être partout dans son royaume pour présider la fête, il lui suffisait d'envoyer son manteau pour le représenter (Conteneau, 1975 : 117). Plus près de nous, chez les Arabes de Moab au début du $20^{\mathrm{e}}$ siècle, la femme stérile empruntait la robe d'une femme qui avait eu plusieurs enfants dans l'espoir d'être tout aussi féconde (Jaussen, 1948: 35). Sachant que le vêtement pouvait être perçu comme un substitut de la personne, ce rite de la déchirure du vêtement pourrait donc être considéré comme un substitut au rite de deuil qui consistait à se taillader le corps et à faire couler le sang, rite qui symbolise par excellence l'acte de se donner la mort (voir section 2).

Troisièmement, la déchirure du vêtement et l'automutilation comme rites de deuil étaient toujours pratiqués en Palestine au début du $20^{\mathrm{e}}$ siècle et le nom qui était donné à cette cérémonie ne laisse planer aucun doute: «les morts enterrent les morts » (Wad-el-Ward, 1907: 112-113).

Quatrièmement, le vêtement est non seulement un substitut de la personne, mais il est aussi signe d'identité. En effet, un changement de vêtement est souvent un indice de changement d'identité. Par exemple, lorsque Élisée, en 1 R 19,19-21 ; 2 R 2,11-22, reçoit le manteau d'Élie, non seulement il entre à son service, mais il acquiert aussi son esprit, sa person- nalité et sa fonction. Selon un vieux rite bien connu dans le monde akkadien, c'est l'acte de couper la frange du vêtement (sissiktaša ibtuq) de son épouse qui indique qu'il y a divorce et que le lien est rompu (Driver et Miles, 1952: 291) ${ }^{5}$. De façon semblable, dans le monde arabe ancien, pour indiquer qu'on dissout un lien d'allégeance, on enlevait une pièce d'habillement et on la jetait loin de soi (Goldziher, 1962: 126). Dans le même sens, déchirer son vêtement symbolise un changement d'identité. Certes, aux yeux d'autrui, c'est une façon de se faire reconnaître comme une personne endeuillée. Toutefois, pour la personne endeuillée, le rite de déchirer ses vêtements symbolise un retour à la nudité, c'est-à-dire la dépossession ${ }^{6}$, l'humiliation', la perte d'identité et la mort ${ }^{8}$. Déchirer ses vêtements est donc un rite qui permet de s'identifier symboliquement au mort.

Cinquièmement, MQ 14b-15b confirme que ce rite permet de se donner symboliquement la mort, car il oblige explicitement les lépreux à se comporter comme des personnes endeuillées et des personnes qui sont retranchées du monde des vivants. Parmi les comportements imposés aux lépreux, qui symbolisent ce retranchement du monde des vivants, figure le port de vêtements déchirés (voir aussi Lv 13,45 et 2 Sam 1,2) et l'obligation de se laisser pousser les cheveux (voir à ce sujet la section 3 ). Il en va de même de l'excommunication qui exclut la personne de la vie avec Dieu et de la vie en société, car les rites qui l'accompagnent sont des rites de deuil: déchirure des vêtements, port de vêtement noir ${ }^{9}$, enlèvement des souliers ${ }^{10}$, etc. (Bābā Meșiâ' 59b; pour d'autres parallèles entre l'endeuillé et l'excommunié, voir Sem 5,11-12.14; 6,11 et MQ 14b-15b). En résumé, en plus de symboliser la douleur et la colère, le rite de la déchirure du vêtement permet d'effectuer un rapprochement symbolique avec le mort.

Par ailleurs, les textes anciens du ProcheOrient, de la Bible hébraïque et de la littérature intertestamentaire ne témoignent pas d'un rituel en tout point identique à celui qui est présenté dans le traité Sem. Par exemple, il semble que le vêtement était à l'origine bel et bien déchiré en deux (2 R 2,12). Aucun des textes anciens ne précise que l'on ne devait déchirer le vêtement qu'au niveau du col. Cette précision n'apparaît que dans le traité $\mathrm{Sem}^{11}$. Il semble donc qu'on ait voulu amoindrir le caractère tragique de ce rite, qui devait aussi s'avérer onéreux ${ }^{12}$.

Quoi qu'il en soit des raisons qui ont conduit les rabbins à préciser que la déchirure devait se faire au niveau du col, cette précision est particulièrement intéressante, car elle permet de mieux comprendre la visée d'un interdit islamique maintes fois repris dans la tradition: "Ils ne sont pas des nôtres ceux qui se frappent les joues, qui déchirent les encolures de leurs vêtements ( ̌́aqqa al-juyūb) et qui profèrent des invocations de l'époque antéislamique» (B 23,23, 36). Cet interdit est repris dans les mêmes termes dans d'autres hadiths (B 23,23,39-40; 51,8,2) et dans le livre des funérailles d'Al-Qastalani (à l'avenir: AQ) : «Le messager de Dieu, rapporte 'Abdullah Ben Masss'oud, a dit: il ne peut pas se réclamer des nôtres celui qui se frappe les joues, déchire les encolures de ses vêtements ( $\check{s} a q q a$ $a l$-juy $\bar{u} b$ ) et profère les invocations de l'époque antéislamique» (AQ 163, ma traduction). Cet interdit apparaît aussi, dans des termes semblables, en B 23,33,38: «Or l'Envoyé de Dieu a désavoué la femme qui, en signe de deuil, crie (al-ṣâliqat), se rase la tête (al-haliqat) ou déchire ses vêtements (al-šâqat)» (voir aussi B 23,41,8; AD 3124-3125 et AQ 165). On retrouve également cet interdit chez AD: "L'envoyé de Dieu a dit: celui qui se rase la tête, crie et déchire ses vêtements n'est pas des nôtres (AD 3124; voir aussi 3125) ${ }^{13}$.

La précision quant à l'endroit où doit se faire la déchirure, soit à l'encolure du vêtement, ne fait aucun doute: l'interdit vise bel et bien une pratique typiquement juive. En imposant un tel interdit, le Prophète avait donc pour objectif de distinguer les musulmans des juifs. Par contre, lorsque l'interdit ne porte pas sur la seule encolure du vêtement, il peut très bien viser des pratiques communes aux anciens sémites et aux tribus arabes. Dans les deux cas, l'interdit vise un objectif commun: empêcher le vivant de se donner symboliquement la mort et de se rapprocher ainsi de la personne décédée et aimée.

Si l'on en croit certains travaux anthropologiques, ce vieux rite de deuil semble avoir survécu à l'interdit. En effet, cette coutume de déchirer ses vêtements était encore attestée au $20^{\mathrm{e}}$ siècle dans certaines tribus arabes du Proche-Orient (Jaussen, 1948: 96; Lipinski, 1969: 31).

Par ailleurs, on a vu que certains hadiths interdisent simultanément trois autres types de comportement: se frapper ou se gifler les joues (daraba al- hudūd ou laṭama al-ḥudūd) (voir aussi B 23,39,1 ; 23,40,1 ; 41,8,2; AQ 163), pousser des cris (șäliqat) et se raser la tête (al-haliqat) (voir aussi AD 3125). Qui plus est, un autre hadith interdit de se mettre de la poussière sur la tête (B 23,34). De tels comportements sont-ils également caractéristiques des rites de deuil juifs? Pour bien répondre à cette question, il convient de les examiner de manière séparée.

\section{L'INTERDIT DE SE FRAPPER}

En ce qui concerne l'acte de se frapper les joues, qui était parfois accompagné du geste de se griffer le visage (Grütter, 1954: 154), il ne semble pas viser une coutume spécifiquement juive. En effet, le rite qui fait couler le sang est attesté aussi bien dans la littérature du Proche-Orient ancien (voir Descente d'Ishtar 
aux enfers, lignes 38-39; Descente d'Inanna aux enfers, lignes 37-38; Ba'al et la mort II, $1 \mathrm{AB}, 2-6)$ que dans la Bible hébraïque (1 R 18,28; Mi 4,14; Jr 16,6; 41,5; 47,5; 48,37). Par contre, ce rite de deuil était formellement interdit dans la Torah (Lv 19,28; 21,5; Dt 14,1), à Qumran (Rouleau du Temple 48,8-10) et dans le Talmud ('Abôdāh Zārāh 17b): «Ne vous faites pas une incision ( $\check{s}$ eret ) dans votre chair pour un mort» (Lv 19,28). D'aucuns voient dans ces automutilations un vieux rite en l'honneur du mort, une sorte de sacrifice rédempteur du fait que la vie réside dans le sang (Lv 17,11.14) (Morgenstern, 1966: 135). Toutefois, dans le monde rabbinique où le monothéisme est de rigueur, ce rite a plutôt une double valeur. Premièrement, il permet d'exprimer une intense douleur, voire une colère retournée contre soi, qui peut éventuellement témoigner d'une certaine forme de culpabilité. Deuxièmement, ce rite est une façon de se rapprocher symboliquement du mort. C'est ainsi par exemple que j'interprète Sem 9,2 qui mentionne que rabbi Aquiba, à la mort de rabbi Eliézer, se frappa sur le cœur (makkeh 'al libô; voir aussi MQ 27b) et que le sang coula ( $w^{e}$ haddām šôtet). Par ailleurs, le Talmud fait aussi référence à l'acte de se frapper les mains de désespoir (MQ 27b.28b) et de frapper du pied par terre (MQ 27b). De tels gestes servaient peut-être à accompagner les lamentations, les chants et la musique (Feldman, 1977: 137).

Des rites semblables sont déjà connus dans les textes du Proche-Orient ancien, la Bible hébraïque et la littérature intertestamentaire: se frapper (sāpad) le cœur (lēb: Nah 2,8; voir aussi Joseph et Aséneth 10,17; Oracles sibyllins 5,193; Vie grecque d'Adam et Ève 42,8), les seins ( $̌$ ădayim Is 32,12 ) ou la cuisse (yārēk: Ez 21,17; Descente d'Ishtar aux enfers, ligne 100). Lorsque Ben Sira, en 38,17, invite à échauffer un coup (thermanon kopeton) en signe de deuil, il fait probablement allusion à l'acte de se frapper la poitrine. Ce geste de se frapper la poitrine en signe de deuil était déjà connu des voisins d'Israël : il est attesté dans le récit de la complainte d'Inanna sur le trépas de Dumuzi (17,15-18), dans un texte d'Hérodote qui évoque les rites de deuil égyptiens (Euterpe 2,85) et dans un passage de Lucien qui décrit un rite de deuil annuel pratiqué par les gens de Byblos (De la déesse syrienne 6). Il a également été remarqué sur le sarcophage du roi de Byblos, Ahirom, qui date entre le $13^{\mathrm{e}}$ et le $10^{\mathrm{e}}$ siècle avant l'ère chrétienne (Sadèr, 1998: 127). En outre, plusieurs stèles funéraires et bas-reliefs égyptiens présentent des femmes en train de se frapper la tête en signe de deuil (Keel, 1997: 319; Erman, 1952: 336 et planche 7). En résumé, l'interdit de se frapper les joues et de s'égratigner le visage - gestes qui miment la mort, gestes de violence retournée contre soi et qui peuvent exprimer aussi bien une grande tristesse qu'une colère - ne vise vraisemblablement pas une pratique juive, mais plutôt une coutume arabe et païenne de la période antéislamique. Si l'on en juge d'après des observations rapportées au $20^{\mathrm{e}}$ siècle, cet interdit islamique semble être resté théorique, puisque ce rite de deuil était toujours existant dans certaines tribus arabes du ProcheOrient (Jaussen, 1948: 96; Lipinski, 1969: 31) et chez certains juifs du Maghreb (Zafrani, 2002: 346).

\section{L'INTERDIT DE SE RASER LA TÊTE}

Le rite qui consiste à se raser la tête en signe de deuil est bien connu dans les textes du Proche-Orient ancien (Baal et la Mort I AB, 6, 18; I AB, 1, 3, Épopée de Gilgamesh $8,2,21$ et Lucien, De la déesse syrienne 6) et dans la Bible hébraïque (Is 3,$24 ; 7,20 ; 15,2$; 22,12 ; Jr 2,16; 7,29; 16,6; 41,5; 47,5; 48,37; Ez 5,1; 7,8; 27,31; Am 8,10; Mi 1,16; Jb 1,20; Esd 9,3), mais il semble n'être attesté qu'une seule fois dans la littérature intertestamentaire (Testament d'Abraham 11,6.11). La disparition de ce rite est sans doute due à l'interdit qu'on trouve en Dt 14,1: «Vous êtes des fils pour Yhwh votre Dieu. Vous ne vous ferez pas des incisions et vous ne placerez pas une tonsure entre vos yeux pour un mort» (Dt 14,1). D'aucuns estiment que cette loi (voir aussi Lv 19,27-28) s'oppose à une ancienne pratique sacrificielle qui visait, d'une part, à rendre un culte au fantôme du défunt et, d'autre part, à le libérer des liens qui l'unissaient à lui (Morgenstern, 1966: 105-106; 145; 225-227; pour l'islam, voir Goldziher, 1967 : 225-227 ). Ce n'est pas impossible, mais aucun texte ancien ne confirme clairement cette interprétation. C'est pourquoi j'entends proposer une autre lecture.

Mais il convient auparavant de signaler que, dans le monde juif, le rite qui consiste à se raser la tête ou à se couper les cheveux semble avoir disparu autour du premier ou du deuxième siècle de l'ère chrétienne, car il apparaît pour la dernière fois dans un passage du Testament d'Abraham qui date du premier siècle et il est absent des traités MQ et Sem. Qui plus est, dans ces deux traités rabbiniques, la règle exige que la personne endeuillée se laisse pousser les cheveux et qu'elle ne puisse se les couper avant les trente premiers jours ou, pour celui qui est en deuil de son père ou de sa mère, après que ses compagnons lui aient fait des remarques désobligeantes sur sa chevelure trop longue et désordonnée, symbole de son retrait de la vie en société à l'instar de l'être aimé qui est décédé (Sem $7,8.11 ; 9,11$; MQ 14a; 14b; 17b; 19b; 22b; $27 \mathrm{~b}$; pour certains règlements concernant la coupe de cheveux, voir Sem 5,10-11).

La coutume de se raser la tête en signe de deuil semble avoir été commune chez les anciens Arabes et les anciens Syriens et elle l'est toujours dans certaines tribus arabes (Morgenstern, 1966: 105-106). Pour bien comprendre le sens de ce vieux rite, il faut savoir que les cheveux étaient le symbole de la force vitale. Le récit de Samson en Jg 13-16 et, plus particulièrement, le texte de Jg 16,17, où Samson dévoile le secret de sa force à Delîlâh, en témoignent avec éloquence: «Le rasoir n'est jamais passé sur ma tête, car je suis consacré à Dieu depuis le ventre de ma mère. Si j'avais les cheveux coupés, alors ma force se retirerait loin de moi, je m'affaiblirais et je serais comme tous les êtres humains. » C'est aussi parce qu'on savait que les cheveux continuaient de pousser après la mort (voir Sem 4,12 ) qu'ils étaient le symbole de la grande vitalité. Les cheveux étaient non seulement une partie essentielle de la personne, mais ils pouvaient même servir de substitut de la personne. C'est, par exemple, le cas en Mésopotamie (Finet 1969: 100-130), mais aussi dans le Coran, où le toupet désigne à la fois la personne entière et sa force vitale, sa vie: «Mais non, s’il ne cesse pas, Nous le trâ̂nerons par le toupet (bi-nnāșiyati); un toupet (nāṣiyatin) menteur et coupable» $(96,15-16)$; «Je m'en remets à Dieu, mon Seigneur et votre Seigneur: point d'être vivant qu'il ne maîtrise par le toupet (bināșiyatihāa). Mon Seigneur dispose du chemin de rectitude» $(11,56)$. Dans le monde arabe ancien aussi bien que moderne, lorsqu'on voulait éviter de tuer son ennemi afin d'empêcher la vengeance du sang, on lui coupait le toupet ou on lui rasait la tête et on le laissait ensuite en liberté (Morgenstern, 1966: 84-86; Chelhod, 1955: 135; Jaussen, 1948: 94 ; 369). Selon cette coutume, couper les cheveux, c'est enlever la force vitale, c'est symboliquement donner la mort à autrui. Un autre rite confirme mon interprétation. Dans l'Arabie ancienne, les guerriers se rasaient parfois la tête avant le combat afin d'indiquer qu'ils se consacraient à la mort (Goldziher, 1967 : 226; Chelhod, 1955 : 136). À mon avis, la vieille coutume sémitique de se raser la tête en signe de deuil visait un objectif semblable: il s'agissait de se donner symboliquement la mort. La loi de Dt 21,12 témoigne, elle aussi, de cette séparation symbolique irrévocable lorsqu'elle prescrit à la prisonnière de guerre qui va se marier de se raser la tête en signe de deuil pour son père et sa mère qui n'ont pas nécessairement été tués. En définitive, tous ces exemples montrent que c'est parce qu'il n'était pas permis à l'endeuillé de se rapprocher symboliquement du défunt que la tradition musulmane a imposé cet interdit de se raser la tête.

\section{L'INTERDIT DE SE METTRE DE LA POUSSIĖRE SUR LA TÊTE}

Cet interdit n'apparaît qu'une seule fois dans toute la section consacrée aux funérailles chez Bukhârî: "'Omar a dit: Laissez les femmes pleurer sur Abou-Solaïmân pourvu qu'elles ne mettent pas de poussière $(t \bar{u} r \bar{a} b)$ 
sur leur tête, ou qu'elles ne poussent pas de cris aigus» (B 23,34).

Ce rite qui consiste à se mettre de la poussière sur la tête en signe de deuil est bien attesté dans les textes du Proche-Orient ancien (Baal et la Mort I AB, VI, 15-16; Légende d'Aqhat A 6,34-38), la Bible hébraïque (1 Sam 4,12; 2 Sam 13,19; 15,32; Is 52,12; 61,3; Ez 27,30; Lm 2,10; Jb 2,12), le premier livre des Maccabées $(1$ Mac 3,$47 ; 4,39 ; 11,71)$ et les textes intertestamentaires (Testament de Job 28,3; 29,4; Martyre d'Isaïe 1,10; 4 Esdras 9,38; Vie grecque d'Adam et Ève 9,3; Paralipomènes de Jérémie 12,1-2; 4,6; 7,20; 9,9; Joseph et Aséneth 10,16 et 13,4). Par contre, bien qu'il n'ait jamais fait l'objet d'une interdiction, ce rite est complètement ignoré du MQ et de Sem. On peut en déduire que ce vieux rite ne faisait déjà plus partie des coutumes juives autour du $3^{\text {e }}$ siècle de l'ère chrétienne.

La signification de ce rite ne pose guère de difficulté. La poussière est traditionnellement associée à la mort, aussi bien dans les textes du Proche-Orient ancien (Gilgamesh $7,4,37 ; 12,96$; La descente d'Ishtar aux enfers $1,8)$ que dans les textes de la Bible hébraïque ('āphār: Gn 3,19; Jb 4,19; 7,21; 10,9; 17,16; 20,$11 ; 21,26$; Ps 22,16.30; 30,10; 44,26; 90,3; 103,$14 ; 104,29$; etc.) et du Coran (turāb: $23,35.82 ; 37,16.53 ; 56,47)$. Par conséquent, se jeter de la poussière sur la tête, c'est vivre symboliquement et temporairement une situation similaire à celle du défunt. En l'interdisant, l'islam refuse donc que l'endeuillé s'identifie symboliquement et par solidarité au défunt qui retourne à la poussière.

\section{L'INTERDIT DE SE LAMENTER}

Les pleurs $\left(b \bar{a} k \bar{a} h^{14}\right)$, les lamentations ('ābal ${ }^{15}$, sāpad ${ }^{16}$, qînāh ${ }^{17}$, nāhāh et nehî $\left.{ }^{18}\right)$, les cris $\left(s \bar{a}^{\prime} a q\right.$ et $\left.z \bar{a}^{\prime} a q 1^{9}\right)$ et les gémissements (yālal: Jr 49,3) en signe de deuil sont bien connus dans la Bible hébraïque. Bien entendu, de telles façons d'exprimer ses émotions face à la mort d'un proche sont universelles et il n'y a pas lieu d'y voir, comme le pense Morgenstern (1966: 145), un rite qui vise à faire peur au fantôme du défunt. Par contre, pleurer, se lamenter et crier ne sont pas que des comportements spontanés de deuil. Ils constituent aussi des comportements ritualisés. En effet, il y avait des pleureurs professionnels ${ }^{20}$ et plus particulièrement des pleureuses professionnelles (2 Sam 1,24; Jr 9,16.19; 49,3; Ez 32,16; Ps 78,64; Jb 27,15). Le Talmud stipule que «même le plus pauvre en Israël ne louera pas moins que deux flûtes et une pleureuse ( $m^{e}$ qônenet) pour les funérailles de sa femme» ( $K^{e}$ tûbôt 46b). Sem 14,7 évoque également le devoir d'avoir deux flûtes et des pleureuses $\left(m^{e} q \hat{o} n^{e} n \hat{o} t\right)$.

Bien que les pleurs et les lamentations ne soient pas interdits dans le judaïsme (MQ 21b; $25 \mathrm{~b} ; 28 \mathrm{~b})$, les rabbins invitent à une certaine retenue: «Ne pleurez pas sur un mort, plus qu'il n'est raisonnable. Et ne vous lamentez pas sur lui, au-delà de toute mesure. Comment cela? Trois jours consacrés aux pleurs. [...] À partir de là, le Saint béni soit-Il dit: Vous n'avez pas à avoir plus de compassion pour lui que Je n'en ai eu Moi-même» (MQ 27b; voir aussi Si 38,16-18). En outre, les prières juives récitées par les personnes en deuil, comme le Qaddîš ou la prière du Șidûq ha-Dîn, «la justification du jugement divin», indiquent clairement que Dieu est toujours reconnu comme une Juge juste (voir Sem 8,12, mais aussi 2,6 et 10,2). Cette reconnaissance trouve peutêtre son origine dans des paroles de Rabbi Hanina ben Teradion, de sa femme et de sa fille, telles que les rapporte le traité 'Abôdāh Zārāh 17b-18a: «Les Romains conduisirent Rabbi Hanina ben Teradion [...] au lieu où il devait être brûlé - sa femme, être tuée - et leur fille, être violée [...]. À leur sortie du tribunal, tous les trois déclarèrent leur soumission au juste jugement de Dieu.»»

Il est donc peu vraisemblable que l'interdit musulman de crier et de proférer des invocations de l'époque antéislamique vise des comportements spécifiquement juifs. $\mathrm{Au}$ contraire, la consigne du Prophète s'apparente plutôt à celle du traité MQ que je viens de citer puisqu'il recommande, lui aussi, trois jours de deuil: «Omm-Atiyya a dit: Il nous était interdit de porter le deuil d'un mort pendant plus de trois jours, à moins qu'il ne s'agît d'un mari et alors le deuil durait quatre mois et dix jours » (B 6,12,1; voir aussi B 23,31,1-3 et AQ 168$)^{21}$. Qui plus est, la tradition musulmane présente à maintes reprises le Prophète ou ses compagnons en train de verser des larmes en signe de compassion: «Plus tard nous entrâmes encore chez Abou-Saïf au moment où Ibrâhîm rendait le dernier soupir. Les yeux du Prophète se mirent à répandre des larmes (tadrifān), et comme 'Abderrahmanben-'Auf lui disait: Toi aussi, ô Envoyé de Dieu!, il répondit: Ô Ibn-'Auf, c'est un effet de la compassion » $(23,44,1$; voir aussi $23,3,1-2$; $23,4,2 ; 23,26,1 ; 23,33,1-2 ; 23,45,1 ; 23,72,1$; AQ 153). Comme dans la tradition rabbinique, certains hadiths invitent à la retenue: " 'Omar a dit: Laissez les femmes pleurer (yabkina) sur Abou-Solaïmân pourvu qu'elles ne mettent pas de poussière sur leur tête, ou qu'elles ne poussent pas de cris aigus » (B 23,34). Selon AQ 168, «il est tolérable de pleurer (buka') sur le mort», mais «sans se lamenter (nawh) ni se déchirer l'encolure du vêtement ( $\check{s} a q$ jayb)». D'autres hadiths vont permettre les pleurs, mais à la condition que l'on pleure en silence (AD 3120; 3165 ; voir aussi B 23,45,1; AQ 153). C'est donc la nature bruyante des lamentations qui est critiquée dans ces hadiths.

Certains hadiths témoignent par ailleurs d'une plus grande intolérance à l'égard des pleurs et des lamentations. L'un d'entre eux signale que le Prophète aurait rétorqué à un homme qui n'arrivait pas à faire taire les femmes qui se lamentaient: «Fourre-leur de la terre dans la bouche» (B 23,41,1)! Dans le même sens, un hadith signale que 'Omar, le compagnon du Prophète, frappait à coups de bâton ceux qui pleuraient, qu'il leur jetait des pierres et leur fourrait de la terre dans la bouche (B 23,45,1)! Selon AQ 153, 'Omar sanctionnait de manière tout aussi violente ceux qui pleuraient: «il ordonnait de les fouetter, de les lapider ou de leur jeter du sable». Puis, commentant ce hadith, AQ ajoute: «Comme le Prophète avait fait avec les femmes de Ja'far. » Il est intéressant de noter que plusieurs des hadiths qui font référence aux pleurs concernent précisément les pleurs d'une femme (B 23,7,1; 23,32,1; 23,33,3-4; $23,35,1$; AD $3118 ; 3122 ; 3124)$ ou de plusieurs femmes (B 23,33,3; 23,34; 23,46,1-2; AD 3105; AQ 153 ; 165). Bien entendu, parmi ces femmes, il y avait des pleureuses professionnelles (Grütter, 1954: 153; Halevi, 2007 : 119). Toutefois, dans le monde arabe antéislamique, contrairement au monde juif, aucun joueur de flûte ou musicien ne semble avoir accompagné ces pleureuses.

Dans certains hadiths, la raison donnée pour justifier la retenue est fort différente: «El-Moghîra a dit: J'ai entendu le Prophète prononcer ces mots : [...] Tout mort sur lequel on pousse des gémissements sera châtié en raison de ces gémissements (nīha)» (B 23,34,1). La même justification est reprise en B 23,34,2: «D'après 'Omar, le Prophète a dit: Le mort sera châtié dans sa tombe en raison des gémissements ( $b i-b \bar{u} k \bar{a}$ ' $i)$ qu'on pousse sur lui.» Maints autres hadiths font allusion à cette croyance selon laquelle les lamentations des personnes endeuillées provoquent les peines et le châtiment du défunt ${ }^{22}$.

Cette croyance très bien attestée dans les hadiths semble avoir été assez populaire, puisqu'elle est rapportée par plus d'une personne. Toutefois, elle pose un sérieux problème, car elle est en contradiction avec l'idée de la responsabilité individuelle, souvent et clairement exprimée dans le Coran:

Redoutez un Jour:

où nul ne sera récompensé pour autrui où nulle intercession ne sera acceptée où nulle compensation ne sera admise où personne ne sera secouru. $(2,48$; voir aussi 6,$164 ; 16,25 ; 29,12 ; 34,25 ; 35,18$; $39,7 ; 53,36-38)$

C'est pourquoi la tradition fait appel à Aïsha pour la combattre:

Plus tard, lorsque 'Omar fut mortellement frappé, Șohaïb entra en pleurant et en criant: Ah! Frère! Ah! Ami! - Ô Șohaïb, est-ce pour moi que tu pleures? demanda 'Omar; or, l'Envoyé de Dieu a dit: Le mort sera châtié pour partie des lamentations (bi-ba'dhi $b \bar{u} k \bar{a}$ ' $i$ ) auxquelles se livrera sa famille à cause de lui. Après la mort de 'Omar, 
je rapportai ces paroles à 'Aïcha qui me dit: Dieu fasse miséricorde à 'Omar! Mais par Dieu! L’Envoyé de Dieu n'a pas enseigné que Dieu châtierait le croyant à cause des pleurs $\left(b i-b \bar{u} k a^{\prime} i\right)$ que verserait sur lui sa famille; il a simplement dit que Dieu accroîtrait le châtiment du mécréant à cause des pleurs ( $\left.b i-b \bar{u} k a^{\prime} i\right)$ versés sur lui par sa famille. Et elle ajouta: Qu'il vous suffise de tenir compte de ces mots du Coran: Aucune âme, chargée de son fardeau, n'aura à supporter le fardeau d'autrui. [voir Coran 35,19] (B 23,33.3) La critique de cette croyance est reprise dans les deux hadiths qui suivent:

Abou-Moussa-'Abdallah-ben-Qaïs a dit : Lorsque 'Omar eut été mortellement frappé, Sohaïb se mit à s'écrier: Ah! Frère!' 'Omar lui répondit: Ne sais-tu pas que le Prophète a dit: Le mort sera châtié à cause des lamentations du vivant? 'Aïcha a dit: Voici simplement ce qu'il en est: L'Envoyé de Dieu, passant près du tombeau d'une juive sur laquelle sa famille pleurait ( $y a b k \bar{\imath})$, s'écria: ces gens-là pleurent (layabkūna) sur elle, et elle, elle sera sûrement châtiée dans sa tombe. (B 23,33,4-5; voir aussi AD 3123) Par ailleurs, pour justifier la retenue dans les lamentations, et a fortiori des rites plus violents mentionnés ci-dessus, la tradition fait unanimement appel à la patience, la soumission et la résignation, trois termes qui traduisent le même mot arabe șabr: «le Prophète passant auprès d'une femme qui pleurait $(t a b k \bar{\imath})$ sur une tombe, lui dit: Crains Dieu et sois résignée (ittaqī llāha wa-ṣbirī) » (B 23,7,1). Maints autres hadiths témoignent de ce que la résignation constitue l'idéal musulman en matière de deuil (voir B 23,32,1; 23,33,1; 23,43,1; AD 3118). Cette patience-soumission, qui s'oppose à la plainte (Coran 14,21), et qui permet de supporter les justes arrêts divins (Coran 52,48; 68,48; 76,24; etc.; voir aussi AD 3105 en lien avec le deuil), constitue la vertu par excellence de l'islam et c'est pourquoi tout musulman doit prier pour l'obtenir (B 24,50,1; voir aussi Coran 2,45 où la patience-résignation est jumelée à la prière). Comme le résume si bien Weinsick, dans les commentaires du Coran, «le concept de șabr, dans toutes ses nuances de sens, est essentiellement hellénistique, car il contient l'ataraxia stoïcienne et la patience chrétienne, la maîtrise de soi et le renoncement ascétique» (1995: 705).

En définitive, même si juifs et musulmans n'ont pas exactement la même conception de ce qui est excessif dans les rites de deuil, les uns et les autres critiquent l'excès car il témoigne d'un manque de foi en la justice divine $^{23}$.

\section{L'INTERDIT DE RETARDER L'ENTERREMENT}

Comme Dieu est celui qui fait vivre et qui fait mourir (Coran 2,258; 23,80; 40,68; voir déjà Dt 32,39; 1 Sam 2,6), la mort ne peut être acceptée qu'avec soumission et résignation. Par conséquent, il est non seulement interdit d'exprimer son deuil par des lamentations excessives et par des rites qui miment la mort, mais il est aussi prescrit d'enterrer les morts rapidement:

D'après Abou-Horaïra, le Prophète a dit: Prenez l'allure rapide en emportant le brancard funèbre. Si le défunt était un homme de bien, il est préférable que vous le fassiez avancer (rapidement) vers le lieu qui l'attend; s'il ne l'était pas, vous déchargerez vos épaules d'une chose malfaisante. (B 23,52,1; voir aussi B 23,91,1; AD 3149; 3153; 3175; 3176; 3178; AQ 256)

L'importance de procéder avec diligence à l'enterrement est bien illustrée dans le récit suivant:

Ibn-'Abbâs a dit: Un homme, que

l'Envoyé de Dieu avait visité (en qualité de malade), vint à mourir. Sa mort ayant eu lieu pendant la nuit, on l'enterra la nuit même. Le matin, on informa le Prophète de l'évènement. Qu'est-ce donc, dit-il, qui vous a empêché de m'aviser plus tôt? -

C'était la nuit, répondit-on, et nous avons craint, en pleine obscurité, de t'infliger quelque peine. Le Prophète se rendit aussitôt sur la tombe et y pria. (B 23,5,1)

Maints autres hadiths indiquent qu'il était si important de faire vite qu'on n'hésitait pas à enterrer le mort même durant la nuit (B 23,70,1; AD 3158). Selon Grütter (1957: 98), cette consigne avait pour objectif de distinguer les musulmans non seulement des païens, mais aussi des juifs et des chrétiens. C'est du moins ce dont témoigne un hadith rapporté par Ibn Sa'd: «Imrân b. al-Husayn ordonna au moment de mourir: Quand après ma mort vous me porterez dehors, avancez vite et ne marchez pas lentement comme les juifs (tahawwada al-yahüd) et les chrétiens. » (Ibn Sa'd VII,1,6, 13). Selon Vajda (2008: 253, note 68 ), qui avoue ignorer si l'allure du convoi était réglée par la coutume ou la législation talmudique, le jeu de mots entre hawwada et yahūd donne à réfléchir sur la valeur documentaire de ce texte.

Or, qu'en est-il de la législation rabbinique? Le traité MQ 22a nous donne deux précieux renseignements à ce sujet:

Pour tous les décès, plus on se hâte de sortir le cercueil [pour l'inhumation], plus on fait œuvre méritoire. Mais pour son père ou pour sa mère, ce serait [au contraire] une conduite condamnable. [Toutefois] la veille d'un Chabbat ou la veille d'une fête, ce serait œuvre méritoire, puisqu'on ne le ferait que par déférence pour son père ou sa mère [sans retarder outre mesure l'inhumation].

Le traité Sem confirme que c'est un devoir d'enterrer les morts rapidement, sauf s'il s'agit d'enterrer ses parents:

Pour tous les autres morts, l'enterrement doit se faire rapidement et les funérailles ne doivent pas être élaborées. Dans le cas de son père ou de sa mère, il doit faire des funérailles élaborées et ne pas se hâter pour l'enterrement, car il est louable de prendre soin de son père et de sa mère. Toutefois, dans des situations d'urgence ou la veille d'un sabbat ou si la pluie va tomber sur le cercueil, il doit se hâter de faire l'enterrement et il ne doit pas prolonger les funérailles. (Sem 9,9)

Selon le traité Sem, l'idéal est également d'enterrer les morts avant la tombée de la nuit, mais on reconnaît qu'il peut y avoir des empêchements :

Quiconque laisse ses morts sans sépulture jusqu'au lendemain leur fait honte. $\mathrm{Si}$, par ailleurs, le retard est dû au fait qu'il faut creuser une tombe ou trouver un linceul ou attendre que les parents arrivent d'un autre endroit, on peut laisser les morts durant la nuit sans aucune préoccupation. (Sem 11,1)

Le traité San'hederîn 6,5 témoigne du même avis : «car toute personne qui laisse un mort (sans sépulture) commet une transgression; cependant, s'il s'agit d'honorer le mort, de lui procurer un cercueil et un linceul, on ne commet pas de transgression». Enfin, le traité MQ nous informe que ceux qui portaient le défunt au cimetière pouvaient s'arrêter en route pour faire une oraison funèbre et, probablement, pour reprendre leur souffle (MQ 27b-28a). L'ensemble de ces informations invite à une conclusion nuancée et prudente: l'idéal des rabbins du Talmud était d'enterrer le défunt rapidement, mais les exceptions étaient assez nombreuses pour susciter la critique de la part des musulmans.

\section{L'INTERDIT DE RESTER DEBOUT LORQUE PASSE UN CONVOI FUNËBRE}

Comment faut-il se comporter lorsque l'on voit passer un convoi funèbre? À cette question, la tradition musulmane a apporté deux réponses. La première prône l'adoption d'une posture verticale: «D'après 'Âmirben-Rabî'a, le Prophète a dit: Lorsque vous voyez passer un convoi funèbre, levez-vous et restez debout jusqu'à ce qu'il vous ait dépassé» (B 23,47,1). Dans ce hadith comme dans plusieurs autres, celui qui s'est levé ne peut se rasseoir que lorsque le convoi l'aura dépassé (B 23,48,1; AD 3166; AQ 166). Quant à ceux qui suivent le convoi, ils ne peuvent s'asseoir avant que le brancard n'ait été posé à terre $(23,48,2 ; 23,49,1$; AD 3166 ; 3167). Cette règle s'applique aussi lorsqu'il 
s'agit du convoi funèbre d'un juif, d'une part parce qu'il s'agit bel et bien d'un être humain (nafsan: B 23,50,1-2), d'autre part parce que la mort est une terreur (AD 3168). Par contre, d'autres hadiths témoignent d'une ouverture moins grande. Par exemple, la raison pour laquelle il faut rester debout lorsqu'un convoi funèbre passe, peu importe que ce soit celui d'un juif, d'un chrétien ou d'un musulman, est qu'il faut marquer un respect, non pour le mort mais pour les anges qui l'accompagnent (Ahmed ben Hanbal, Musnad Ahmed 4,391, cité dans Vajda, 2008: 238). Un autre hadith, décidément plus polémique à l'égard des juifs, rapporte que le musulman doit se lever au passage du convoi d'un juif décédé parce qu'on ne devrait pas permettre aux juifs de marcher la tête placée plus haut que celle d'un musulman (Al-Kulayni, al-Kâfî, KJ, 192, cité dans Halevi, 2007: 158).

Cependant, un hadith rapporté par AD est équivoque à ce sujet: " 'Alî b. Abî Tâlib a dit: Le Prophète se tenait debout pour les funérailles et après cela il s'asseyait» (AD 3169). Dans la note qui accompagne ce hadith, le traducteur (Hasan, cité dans Dawud, 1988: 901) rappelle que les commentateurs sont partagés sur la façon dont il faut comprendre la proposition temporelle «et après cela»: d'aucuns croient que le Prophète ne s'est assis qu'une fois que le convoi funèbre fut passé, tandis que d'autres estiment que le Prophète abroge ici une coutume pour en proposer une nouvelle. À mon avis, cette seconde interprétation semble celle qui est défendue par $\mathrm{AD}$, puisque le hadith qu'il rapporte ensuite indique clairement que le Prophète abroge l'ancienne coutume afin de bien distinguer les musulmans des juifs: «'Ûbâdah b. al Șâmit a dit: L'Envoyé de Dieu avait l'habitude de rester debout lors des funérailles jusqu'à ce que le mort fut mis dans la tombe. Un juif érudit passa et lui dit: C'est ce que nous faisons. Le Prophète s'assit et dit: Assoyez-vous et ne faites pas comme eux.» (AD 3170). Rien dans les traités MQ et Sem ne permet de confirmer ou d'infirmer cette affirmation. Juste ou pas, ce hadith, qui a fini par abroger l'ancienne pratique, indique par la négative l'étroite parenté qui unissait judaïsme et islam à l'origine.

\section{POUR NE PAS CONCLURE...}

Plusieurs autres interdits auraient pu être étudiés, mais mon analyse comparée de sept interdits, bien que partielle, me permet de dégager quelques dissimilitudes et similitudes significatives entre les règles provenant des hadiths de l'islam sunnite et celles du judaïsme rabbinique.

Examinons d'abord les dissimilitudes. J'ai montré que les musulmans cherchent à se distinguer des juifs dans au moins trois cas: l'interdit de déchirer l'encolure de son vêtement, de retarder l'enterrement et de rester debout lorsque passe un convoi funéraire.
Dans le cas du rite de la déchirure (de l'encolure) de son vêtement, l'interdit vise les juifs, même si ceux-ci ne sont pas explicitement nommés, mais aussi les païens. L'interdit de retarder l'enterrement vise explicitement les juifs, mais également les païens et les chrétiens. Par contre, l'interdit de rester debout lorsque passe un convoi funéraire ne vise explicitement que les juifs. L'histoire de cet interdit est intéressante, car elle témoigne essentiellement d'une volonté ferme de se distinguer des juifs. En effet, c'est le seul cas où le Prophète abroge une pratique qui était auparavant observée par les premiers musulmans. C'est aussi le seul rite interdit qui n'a guère de signification religieuse profonde.

Par contre, les autres interdits découlent de motifs qui vont bien au-delà du simple désir de vouloir se distinguer des autres groupes socioreligieux. En effet, j'ai montré que plusieurs des rites interdits visaient à rapprocher symboliquement le vivant du mort. C'est le cas des rites suivants: l'automutilation, la déchirure (de l'encolure) de son vêtement, le rasage des cheveux et le jet de poussière sur la tête. À mon avis, c'est parce que ces rites permettaient aux vivants de s'identifier symboliquement au défunt qu'ils ont été interdits. En effet, dans l'islam, les rites autour de la mort sont plutôt axés sur la vie que sur la mort. À l'interdit imposé au vivant de mimer la mort correspond donc en quelque sorte l'obligation de traiter le mort comme un vivant. En effet, dans les hadiths de la tradition sunnite, on insiste beaucoup sur le fait que le mort doit être minutieusement lavé, parfumé, peigné et habillé (B 23,8,1 à 23,29,1; AD 3114; 3136-3152). Les hadiths montrent également que c'est parce que le mort est considéré comme un vivant qu'il est interdit de retarder son enterrement:

D'après Abou-Sa‘îd-El-Khodry, l'Envoyé

de Dieu a dit: Lorsque le cadavre a été

posé sur le brancard, que les hommes

l'ont chargé sur leurs épaules, si le défunt a été un juste, son âme criera: Faites-moi avancer. Dans le cas contraire, l'âme dira: Malheur à moi! Où m'amenezvous? Ces paroles seront entendues par toutes les choses, sauf par l'homme, car l'homme qui les entendrait tomberait foudroyé. (B 23,51,1 ; voir aussi 23,91,1 ; AD 3175-3178)

En outre, il ne convient pas de retarder le jugement de la tombe. Maints hadiths soulignent que l'interdit de se lamenter bruyamment repose, lui aussi, sur la conviction que le défunt, même enterré, entend les lamentations qui sont funestes pour lui (B 23,68,1; 23,87,6; AD 3123; voir aussi B 23,87,2-3). Non seulement le défunt entend le moindre claquement des sandales (B 23,87,6; AD 3225), mais il peut même éprouver un soulagement si une personne plante une branche de palmier verte sur sa tombe (B 23,82,1) ${ }^{24}$. C'est aussi pourquoi un hadith rapporte la prière suivante: «Ô
Dieu, que sa tombe soit spacieuse pour lui et accorde lui de la lumière en elle» (AD 3112). L'interdit de briser les os du défunt repose sur la même conviction: «Selon Aisha, l'Envoyé de Dieu a dit: Briser les os d'un homme mort, c'est comme les lui briser de son vivant » (AD 3201).

Certes, les textes rabbiniques n'ignorent pas l'existence de certains rites axés sur la vie, comme le lavage et l'habillage du défunt. Toutefois, la ritualité juive autour de la mort est beaucoup plus ambivalente, car les textes prescrivent également de nombreux autres rites qui visent à se rapprocher symboliquement du mort. C'est ce que montrerait une étude exhaustive des nombreux rites prescrits aux endeuillés dans les textes rabbiniques. Par exemple, le devoir de renverser son lit symbolise le renversement du cycle de la vie au moyen de l'inversion du lit sur lequel les êtres humains sont conçus (Sem 6,1; 10,10; 11,11$19 ; 12,4$; MQ 15b; 20a; 20b; 21a; 26b-27a). Il est interdit de se préparer à manger, car c'est une action qui ne peut être posée que par une personne bien en vie; il faut aussi consommer certains aliments qui évoquent la mort, comme les lentilles qui n'ont pas de bouche, à l'image de l'endeuillé qui doit rester silencieux et qui doit s'abstenir de questionner son destin (Sem 2,6; 10,3 ; 11,2; 12,4 ; 14,13-14; MQ 23b; 27b; Bābā' Bat' rā' 16b). L'obligation de s'asseoir par terre a pour objectif de rappeler que l'on vient de la terre et permet à la personne endeuillée de se rapprocher symboliquement du défunt (MQ 27a). Les interdits temporaires (la durée varie selon les interdits) de se couper les ongles avec un coupe-ongle (Sem 7,11.13; MQ 17b-18a), de se laver (Sem 6,1; MQ 15b; 21a), de se parfumer (MQ 21a; 27a), de laver ses vêtements (MQ 15a; 17b), de porter des souliers (Sem 6,1; MQ 15b;20b; 21a) et les phylactères (MQ 15a; 21a), de travailler (Sem 5,3-9; MQ 11b; 15b; 21b; 22b; 27b), de lire et d'étudier (Sem 6,1; MQ 15a; 21a), de dire la prière après le repas ou de s'associer à la prière d'autres personnes (MQ 23b), de saluer autrui (MQ 15a; 21b), d'avoir des relations sexuelles (MQ 15b; 24a), etc., sont autant d'interdits qui portent sur des gestes qui caractérisent les seuls vivants. Comme le dit si bien MQ 23a à propos de l'interdit de sortir de chez soi les deux premières semaines de deuil et de l'interdit de s'asseoir à sa place et de parler à la synagogue lors de la quatrième semaine, «ce n'est qu'à la cinquième semaine que l'on redevient comme tout le monde», c'est-à-dire comme des êtres vivants normaux. Bref, contrairement aux textes de la tradition sunnite qui promeuvent un rituel minimaliste et essentiellement axé sur la vie, les textes rabbiniques prescrivent de très nombreux rites qui visent à rapprocher symboliquement le vivant du mort.

Ces nombreuses dissimilitudes entre musulmans et juifs ne doivent pas cacher 
pour autant certaines similitudes. juifs et musulmans, à des périodes différentes de leur histoire, ont tenu à se distinguer des païens. Par exemple, les uns comme les autres ont tenu à se distinguer des païens en s'abstenant de pratiquer les mêmes rites de deuil: se raser la tête et se mettre de la poussière sur la tête. En outre, juifs et musulmans s'entendent pour réprouver l'excès dans les lamentations. Il est vrai que les règles juives et musulmanes ne délimitent pas de la même manière ce qui est excessif et que les règles musulmanes semblent plus strictes que les règles juives - peut-être à cause de la croyance aux châtiments de la tombe? -, mais dans les deux cas le refus de l'excès est ultimement fondé sur une conception théologique semblable: la justice divine. De la même façon, juifs et musulmans s'entendent pour interdire tout geste de violence contre soi. Enfin, juifs et musulmans ont un dernier point en commun que même l'exégète qui ne travaille que sur des textes se doit de rappeler: en divers moments de l'histoire et dans diverses régions du monde, les uns comme les autres ont transgressé ces interdits. Je l'ai souligné par exemple pour les rites de la déchirure des vêtements, de l'automutilation, du rasage de la tête, du jet de poussière sur la tête et des lamentations bruyantes. Il ne faut donc jamais oublier que les frontières religieuses sur le terrain sont toujours plus floues que celles qui sont inscrites dans les vieux textes qui ont été composés pour des personnes vivant dans des contextes socioreligieux très différents $\mathrm{du}$ nôtre. À mon avis, il est heureux qu'il en soit ainsi, car s'il est important de faire dialoguer les vieux textes entre eux, il est encore plus important d'ouvrir ce dialogue aux personnes de diverses croyances qui vivent aujourd'hui, car seul un tel dialogue permet, d'une part, de faire de fructueuses rencontres au-delà des frontières établies et, d'autre part, d'identifier les véritables motifs de ceux qui se donnent aujourd'hui le rôle de douaniers.

\section{BIBLIOGRAPHIE}

\section{Sources}

ALAND, KURT et al. (1966). The Greek New Testament, Stuttgart, Württemberg.

AL-BUKHĀRĪ (1427 / 2007). Sahịh Al-Bukhārī, Al-Qahirat, Maktabat Mașr.

AL-QASTALANI (1993). Jawaher al-Bokhari (Les perles d'Al-Bokhari) et l'interprétation d'Al-Qastalani. Les 700 hadits commentés, traduit par N. Ounes, Beyrouth, dar el Fiker.

BOTTÉRO, JEAN (1992). L'Épopée de Gilgamesh, Paris, Gallimard.

BOTTÉRO, JEAN et SAMUEL NOAH KRAMER (1989). Lorsque les dieux faisaient l'homme. Mythologie mésopotamienne, Paris, Gallimard.
CAQUOT, ANDRÉ et al. (1974). Textes ougaritiques. Tome 1. Mythes et légendes, Paris, Cerf.

CAQUOT, ANDRÉ et al. (1989). Textes ougaritiques. Tome 2. Textes religieux et rituels, Paris, Cerf.

CHELHOD, JOSEPH (1955). Le sacrifice chez les Arabes. Recherches sur l'évolution, la nature et la fonction des rites sacrificiels en Arabie occidentale, Paris, Presses universitaires de France.

DAWUD, SUNAN ABU (1988). English Translation Explanatory Notes by Ahmad Hasan, volume II, chapitres 519-1337, Lahore, Sh. Muhammad Ashrat.

DIODORE DE SICILE, BIBLIOTHÈQUE HISTORIQUE (1993). Introduction générale par François Chamoux et Pierre Bertrac, texte établi par Pierre Bertrac et traduit par Yvonne Vernière, Paris, Les Belles Lettres.

DUPONT-SOMMER, ANDRÉ ET MARC PHILONENKO (DIR.) (1987). La Bible. Écrits intertestamentaires, Paris, Gallimard.

EL-BOKHÂRI (1977). Les traditions islamiques, traduites de l'arabe avec notes et index par O. Houdas et W. Marçais, Tome 1 à 3, Paris, Librairie d'Amérique et d'Orient.

ELLIGER KARL ET WILHELM RUDOLPH (1977). Biblia Hebraica Stuttgartensia, Stuttgart, Deutsche Bibelgesellschaft.

GOLDSCHMIDT, LAZARUS, HRSG. (19331935). Der babylonische Talmud mit einschluss der vollstaendigen Mišna, Haag, Nijoff.

HÉRODOTE, HISTOIRES. LIVRES II. EUTERPE (1948). Texte établi et traduit par Ph. Le Grand, Paris, Les Belles Lettres.

LABAT, RENÉ et al. (1970). Les religions du Proche-Orient asiatique, Paris, Fayard \& Denoël.

LE SAINT CORAN ET LA TRADUCTION EN LANGUE FRANÇAISE DU SENS DE SES VERSETS, révisé et édité par La Présidence Générale des Directions de0s Recherches Scientifiques Islamiques de l'Ifta, de la Prédication et de l'Orientation Religieuse (1410 de l'Hégire). Al-Madinah Al-Munawwarah, Presses du Complexe du Roi Fahd.

LE TALMUD. TRAITÉ MOED KATAN (1988). Traduit de l'hébreu et de l'araméen par Israel Salyer, Lagrasse, Verdier.

LUCIEN DE SAMOSATE (1933) Euvres complètes, Tome 3, traduction nouvelle avec notices et notes par Émile Chambry, Paris, Garnier.

MASSON, DENISE (1967). Le Coran, Paris, Gallimard.

PRITCHARD, JAMES B. (1973). The Ancient Near East: An Anthology of Texts and Pictures, Princeton, Princeton University Press.

RAHLFS, ALFRED (1935). Septuaginta, Stuttgart, Deutsche Bibelstiftung Stuttgart.

SUNAN ABU DAWUD (1988). English translation with explanatory notes by Ahmad Hasan, Volume II - (Chapters 519-1337), Lahore, Sh. Muhammad Ashraf.

THE BABYLONIAN TALMUD (1935-1952). Londres, The Soncino Press.
THE TRACTATE MOURNING ŚĔMAHOT (REGULATIONS RELATING TO DEATH, BURIAL, AND MOURNING) (1966). Translated from the Hebrew with Introduction and Notes by Dov Zlotnick, New Haven - Londres, Yale University Press.

\section{Études contemporaines}

CASSIN, ELENA (1968). La splendeur divine, Paris, Mouton et Co.

COHN-SHERBOF, DAN (1992). The Blackwell Dictionary of Judaica, Oxford, Blackwell.

CONTENEAU, GEORGES (1975). La civilisation d'Assur et de Babylone, Genève, Famot.

DRIVER G. R. et J. C. MILES (1952). The Babylonians Laws, Volume I, Oxford, Clarendon Press.

ERMAN, ADOLPHE (1952). La religion des Égyptiens, Paris, Payot.

FELDMAN, EMANUEL L. (1977). Biblical and Postbiblical Defilement and Mourning: Law as Theology, New York, Yeshiva University Press \& Katv Publishing House.

FINET, ANDRÉ (1969). «Les symboles du cheveu, du bord du vêtement et de l'ongle en Mésopotamie», dans Eschatologie et cosmogonie, Bruxelles, Éditions de l'Institut de sociologie: 100-130.

GOLDZIHER, IGNAZ (1962). Études islamologiques d'Ignaz Goldziher, Traduction analytique par G.-H. Bousquet, Leiden, Brill.

GOLDZIHER, IGNAZ (1967). Muslim Studies, translated from the German by C. R. Barber \& S. M. Stern, Volume I, Londres, George Allen \& Unwin LTD.

GRÜTTER, IRENE (1954). «Arabische Bestattungsbräuche in frühislamischer Zeit (nach Ibn Sa'd und Buhāāī)», Der Islam 31 : 147-173.

GRÜTTER, IRENE (1957). «Arabische Bestattungsbräuche in frühislamischer Zeit (nach Ibn Sa'd und Buhāāī) (1. Fortsetzung)», Der Islam 32: 79-104.

GRÜTTER, IRENE (1957). «Arabische Bestattungsbräuche in frühislamischer Zeit (nach Ibn Sa‘d und Buhārīi) (SchluB)», Der Islam 32: 168194.

GUIRAUD, MORGAN (2007). «Barzakh», dans Mohammad Ali Amir-Moezzi (dir.), Dictionnaire $d u$ Coran, Paris, Robert Laffont: 114-119.

HALEVI, LEOR (2007). Muhammad's Grave. Death Rites and the Making of Islamic Society, New York, Colombia University Press.

JAUSSEN, ANTONIN (1948). Coutumes des Arabes au pays de Moab, Paris, Librairie d'Amérique et d'Orient.

KEEL, OTHMAR (1997). The Symbolism of the Biblical World. Ancient Near Eastern Iconography and the Book of Psalms, Indiana, Eisenbrauns. 
LAVOIE, JEAN-JACQUES (2011). «La légitimation religieuse du rite d'inhumation dans le Coran et la littérature du Proche-Orient ancien. Analyse historico-comparative", dans Khadiyatoulah Fall et Mamadou Ndongo Dimé (dir.), La mort musulmane en contexte d'immigration et d'islam minoritaire: enjeux culturels, religieux, identitaires et espaces de négociations, Québec, Presses de l'Université Laval: 91-113.

LEISTEN, THOMAS (1990). «Between Orthodoxy and Exegesis: Some Aspects of Attitudes in the Shari'a Toward Funerary Architecture», Muqarnas 7: 12-22.

LIPINSKI, EDOUARD (1969). La liturgie pénitentielle dans la Bible, Paris, Cerf.

MORGENSTERN, J. (1966). Rites of Birth, Marriage, Death and Kindred Occasions Among the Semites, Cincinnati, Hebrew Union College Press.

MÜLLER, MAX (1873). Introduction to the Science of Religion: Four Lectures Delivered at the Royal Institution in February and May 1870, Londres, Longmans Green and Co.

RĀGIB, YŪSUF (1992) «Structure de la tombe d'après le droit musulman », Arabica 39: 393403.

SADÈR, HÉLÈNE (1998). «La malédiction d'Ahirom, roi de Byblos", dans Liban. L'autre rive. Exposition présentée à l'Institut du monde arabe du 27 octobre 1998 au 2 mai 1999, Paris, Flammarion: 126-127.

TRITTON, A. S. (1977). «Djanāza», Encyclopédie de l'islam, Paris - Leyde, Maisonneuve \& Larose-E.J. Brill: 453-454.

VAJDA, GEORGES (2008). «Jews ans Muslims according to the Hadith», dans Andrew G. Bostom (dir.), The Legacy of Islamic Antisemitism. From Sacred Texts to Solemn History, New York, Prometheus Books : 235-260

WAD-EL-WARD, GAMAHLIEL (1907). Palestine through the Eyes of Natives, New York, Flemming H. Revell Company.

WALFISH, ABRAHAM (1997). «Ebel Rabbati», dans R. J. Zwi Werblowsky \& Geoffrey Wigoder (dir.), The Oxford Dictionary of Jewish Religion, Oxford, Oxford University Press : 240.

WENSINCK, A. J. (1995). «şabr», Encyclopédie de l'islam, Tome 8, Leiden, Brill: 705707.

ZAFRANI, HAÏM (2002). juifs d'Andalousie et du Maghreb, Paris, Maisonneuve et Larose.

ZAMAN, MUHAMMAD QASIM (2001). «Death, Funeral Processions, and the Articulation of Religious Authority in Early Islam», Studia Islamica 93 : 27-58.

\section{Notes}

1. Avec le beged, «vêtement» en général ou «manteau », voir Gn 37,29; Lv 10,6;21,10; Nb 14,6; Jg 11,35; 2 Sam 1,$11 ; 3,31 ; 13,31 ; 1$ R 21,27; 2 R 2,$12 ; 5,7-8 ; 6,30 ; 11,14 ; 18,37 ; 19,1$; $22,11.19$; Is 37,1 ; Jr 36,24; 41,5; Jl 2,13; Est 4,1; Esd 9,3; 2 Chr 23,13; 34,27. Avec śimelāh, «vêtement de dessus», voir Gn 37,34; 44,13; Jos 7,6); avec $\mathrm{mad}$, «tunique», «robe», voir 1 Sam 4,12; avec $k^{e}$ tonet hapassîm, «tunique à manches», voir 2 Sam 13,19; avec $k^{e}$ tonet, «tunique», voir 2 Sam 15,32; avec $m^{e}$ ' $i l$, «man- teau », voir Jb 1,20 et 2,12; Esd 9,3; sans aucune précision, voir Qo 3,7.

2. Voir Jdt 14,16.19; Lettre de Jr 1,30; 1 Mac 2,14; 3,$47 ; 4,39 ; 5,14 ; 11,71 ; 13,45 ; 2$ Mac 4,38.

3. Jubilées 43,8; Testament de Zabulon 4,5; Testament de Joseph 5,2; Testament de Moïse 11,1; Martyre d'Isaïe 1,10; Livre des Antiquités bibliques 46,4; 54,4; 4 Esdras 9,38; 2 Baruch 9,2; Testament de Job 19,2; 28,3; 39,7; Paralipomènes de Jr 2,1-2.5.8; 9,9; Apocalypse d'Élie 2,21. Le texte de J1 2,13 et celui des Paralipomènes de Jr 2,5 sont très intéressants. En invitant à déchirer les cœurs au lieu des vêtements, ils rappellent que le rite ne signifie rien s'il n'est pas accompagné d'une véritable conversion et de sentiments de douleur.

4. En Mt 26,65, le grand prêtre déchire ses vêtements en signe de deuil, car il estime que Jésus a blasphémé. Il est bien connu que le grand prêtre n'avait pas le droit de déchirer ses vêtements en signe de deuil privé (Lv 21,10; Sem 4,12). Toutefois, selon San ehederîn 7,5, lors d'un blasphème, les juges (hadayyānîm) devaient se lever, déchirer leurs vêtements ( $q \hat{o}^{r}{ }^{\text {în }}$ ), sans jamais les recoudre.

5. Les auteurs rappellent également que la frange du vêtement, qui représentait l'ensemble du vêtement, était aussi imprimée sur les tablettes d'argile afin de signifier que le propriétaire était lui-même personnellement tenu de respecter le contrat qu'il avait établi avec une autre personne.

6. Lorsque David coupe le pan du vêtement de Saül (1 Sam 24,5-6), il accomplit un acte symbolique: Saül devra perdre tout ce qu'il possède, sa royauté et même sa vie. C'est d'ailleurs ce que confirme 1 Sam 24,21. De la même façon, lorsque Jonathan se dépouille de son manteau et de ses armes pour les donner à David, c'est pour lui signifier qu'il lui concède le trône auquel il avait droit (1 Sam 18,4).

7. Voir Dt 28,48; Is 20,2-4; Am 2,16; Mi 1,8; 2 Chr 34,27 . Il n'est pas inopportun de rappeler que le mot humiliation est de la même famille que le mot humus, cette terre qui est le lieu ultime de rendez-vous des vivants!

8. Voir Jb 1,21; Qo 5,14. Lorsque Ishtar descend en enfer, elle est dépouillée de ses vêtements au fur et à mesure qu'elle franchit les sept portes. Lorsqu'elle arrive en enfer, sa mort est symbolisée par sa totale nudité. Par contre, lorsqu'elle remonte de l'enfer pour revenir à la vie, elle reprend ses vêtements (Descente d'Ishtar aux enfers, lignes 1-135).

9. La couleur noir comme symbole de deuil n'est pas inconnue dans la Bible, puisque le verbe qādar signifie à la fois «s'assombrir», «s'obscurcir», «être noir»et «être en tenue de deuil» (Ps 35,14; Jr 4,28; 14,2; Ez 31,15; voir aussi Jb $5,11$ et 30,28$)$. Quant au vêtement noir comme symbole de deuil, il est connu du Talmud (Bābā'

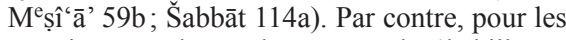
premiers musulmans, la coutume de s'habiller en noir appartient à l'époque de la jāhiliyya (Goldziher, 1967: 235).

10. Ne pas avoir de souliers, c'est être comme un mort (Šabbāt 152a) ou un excommunié ( $\mathrm{P}^{\mathrm{e}}$ sāḥîm $113 b)$. Du côté de l'islam, un hadith rapporte que le Prophète a interdit de marcher entre les tombes avec ses souliers (AD3224). Halevi signale que Ali soutenait qu'il fallait marcher pieds nus derrière un cercueil; c'était là une façon de signaler l'absence d'arrogance et de vanité (2007: 307, note 30 ).

11. MQ témoigne d'une pratique qui semble à michemin entre celle de la Bible hébraïque où le vêtement est déchiré en deux, et celle de Sem où la déchirure se fait à l'encolure du vêtement. En effet, le vêtement peut être déchiré jusqu'au cœur (MQ 22b) ou la déchirure peut être de la largeur d'un empan, c'est-à-dire de la largeur de toute la main, les doigts bien écartés (MQ 26b). Par ailleurs, Sem 9,6 garde la trace de la pratique favorisée par le traité MQ, puisqu'il règlemente ainsi la longueur de la déchirure: elle doit mesurer «trois largeurs de doigt, mais le vêtement ne doit pas être déchiré au-delà du nombril».

12. La modification de ce rite a dû se faire autour de la fin du $3^{\mathrm{e}}$ siècle ou au $4^{\mathrm{e}}$ siècle. C'est peutêtre ce qui explique que le port du sac, qui suivait le rite de la déchirure des vêtements (voir Gn 37,34; 2 Sam 3,31; 1 R 20,31; 21,27; 2 R 6,30 ; 19,1-2; etc.) ne fait l'objet d'aucune règlementation dans le traité Sem. Ce rite n'est mentionné que dans Sem 8,8-9, deux brefs textes qui rappellent que certains rabbins se revêtirent du sac à la mort de Rabbi Aquiba (environ en 135 de l'ère chrétienne). Il semble donc que ce rite, encore attesté dans quelques textes de la littérature intertestamentaire (Psaume de Salomon 2,20; Martyre d'Isaïe 2,10; Testament de Joseph 15,2; Joseph et Aséneth 10,11.16;13,3;14,12), soit disparu durant la période rabbinique, sans doute au même moment où le rite de la déchirure se limita à l'encolure du vêtement. De nos jours, cette loi rituelle de la déchirure n'est plus imposée par tous les rabbins, afin que nul n'évite d'assister un mourant dans ses derniers instants. Certaines personnes arrachent simplement un bouton de leur chemise afin que celle-ci bâille et apparaisse comme déchirée. D'autres achètent la pièce de vêtement (cravate pour l'homme et foulard pour la femme) qui sera déchirée peu avant l'enterrement.

13. Il convient de rappeler que cet interdit de déchirer son vêtement n'existe pas dans l'islam shī'ite. Au contraire, les hommes ont tendance à déchirer leur longue chemise au moment de la mort d'un fils, d'un père ou d'un imam (Halevi, 2007: 293, note 32 ).

14. Voir Dt 21,$13 ; 2$ Sam 1,11-12; 3,32-34; Is 15,2-5; 22,12; Jr 22,10; Ez 27,31; Est 4,1-3; etc. Pour les textes grecs, voir Si 38,16-17 et 1 Mac 9,20.

15. Par exemple, au qal, ce verbe signifie à la fois «être en deuil» et «se lamenter». Voir Is 3,26 ; 19,$8 ; 24,4.7 ; 33,9 ;$ Jr 4,28; $12,4.11 ; 14,2$; 23,10 ; Os 4,3; 10,5; Jl 1,9-10; Am 1,2; 8,8; 9,$5 ; \mathrm{Jb} 14,22$.

16. Voir Gn 23,$2 ; 50,2 ; 1$ Sam 25,$1 ; 28,3 ; 2$ Sam 1,$12 ; 11,26$; $1 \mathrm{R} 13,29-30 ; 14,13.18$; Is 32,12 ; Jr 4,$8 ; 16,4.5 .6 ; 22,18 ; 25,33 ; 34,5 ; 49,3 ; \mathrm{Ez}$ 24,16.23; Mi 1,8; Za 7,5; 12,10.12; J1 1,13; Qo 12,5 .

17. Voir Jr 9,9-10.19-20; Ez 26,17; 27,2.32; 32,2.16; Am 8,10; 2 Chr 35,25; voir aussi 2 Sam 1,17; Jr 7,29; Ez 2,10; 19,1.14; 28,12; Am 5,1 .

18. Voir 1 Sam 7,2; Ez 32,18; Mi 2,4 et $\mathrm{Jr}$ $9,9.17 .18 .19 ; 31,15$; Am 5,16 et Mi 2,4. 
19. Par exemple, avec ș'q, voir Jr 49,3; avec z'q, voir 2 Sam 19,5; Ez 27,30; voir aussi Ex 11,6 et 12,30 .

20. Voir Am 5,16: «ceux qui connaissent la plainte (nehî)»; Qo 12,5: «et tourneront dans la rue ceux qui font la lamentation (hassôp $p^{e-}$ dîm)»; Jb 30,31: «ma harpe est accordée aux chants de deuil, ma flûte à la voix des pleureurs (bokîm)»; 2 Chr 35,25: «tous les chanteurs et les chanteuses dans leurs lamentations (qînôt)».

21. La durée du deuil dans le cas du décès d'un mari a une fonction pratique: il s'agit de s'assurer que la femme ne soit pas enceinte avant qu'elle ne se remarie. Par ailleurs, un hadith rapporté par Anas-ben-Mâlik affirme que le Prophète resta un mois dans la retraite en signe de deuil (B $23,41,2)$. En ce qui concerne le judaïsme rabbinique, la durée du deuil est répartie sur quatre périodes distinctes: entre la mort et l'enterrement, les sept jours qui suivent l'enterrement, les trente jours après l'enterrement et la première année.
22. B $23,45,1$; AQ $153 ; 162$; Voir aussi certains hadiths qui affirment que le défunt qui vient d'être enterré est toujours conscient de ce qui se passe (B 23, 87,2 ; 23,87,6; AD 3225; AQ 257). Ces affirmations ne sont pas sans lien avec la croyance au châtiment de la tombe ('adhāb alqabr) qui est également attesté dans les hadiths (B 23,82,1;23,87,4; 23,881-3; AD 3196; 3215; AQ 168). Fait intéressant, l'un de ces hadiths rapporte cette croyance dans la bouche d'une juive $(\mathrm{B} 23,82,4)$ et l'autre affirme que ce sont des juifs qui subissent ce châtiment dans leurs tombeaux (B 23,88,1). Or, cette croyance dans le châtiment de la tombe - en hébreu : hibût haqqeber - est également connu du monde juif kabbalistique du bas Moyen Âge, particulièrement dans le Yalkût ha rô 'îm. Dans les deux cas, le défunt reçoit d'abord la visite de deux anges (Munkar et Nakīr dans l'islam et un ange de service et un ange de la mort dans le monde juif) qui sont chargés de l'interrogatoire du tombeau. Dans les deux cas, le défunt peut expérimenter à l'avance, selon les réponses qu'il donne, une sorte d'enfer. Pour des informations supplémentaires sur le jugement de la tombe dans l'islam, voir Guiraud (2007: 114-119).

23. Par ailleurs, en ce qui concerne les lamentations, l'islam shī'ite se distingue nettement de l'islam sunnite. Par exemple, d'aucuns affirment même que le shī'isme est le prototype de la religion de la lamentation (Halevi, 2007: 133).

24. Plusieurs autres hadiths témoignent aussi de cette pratique qui remonte à la période antéislamique (Grütter, 1957: 173). 